\title{
高孔率泡沫金属的孔结构保形铁削加工研究
}

\author{
周伟 ${ }^{1}$ 刘阳旭 ${ }^{1}$ 褚旭阳 $^{1}$ 陈 露 ${ }^{2}$ \\ (1. 厦门大学机电工程系 厦门 361005; \\ 2. 哈尔滨工业大学机电工程学院 哈尔滨 150006)
}

\begin{abstract}
摘要: 为避免高孔率泡沫金属的孔结构在加工过程中变形, 提出一种利用填充材料进行泡沫金属固化后再进行孔结构保 形铁削加工的新方法。首先对不同填充材料的泡沫铜进行铣削加工试验, 然后利用 SEM 图进行表面形貌分析, 选用孔 径保形率、孔崉保形率以及透气性来评价泡沫金属铣削加工的保形效果。基于中心组合试验, 以孔径大小和 $0.7 \mathrm{kPa}$ 压 力下的流量值为指标, 建立二次回归方程并计算出在选定填充材料条件下的泡沫铜的优化加工参数。研究结果表明, 以 氢化松香-硬脂酸钠按质量比 4:1 混合的固化填充材料可以使得泡沫铜在铣削加工过程中获得较好的保形效果。孔径保 形率、孔崉保形率以及透气性性能均随主轴转速、进给速度呈先增加后降低的趋势。同时, 在选择优化填充材料的条件 下, 主轴转速为 $311 \mathrm{r} / \mathrm{min}$, 进给速度为 $165 \mathrm{~mm} / \mathrm{min}$ 时, 铣削加工泡沫铜具有较好的保形效果, 其孔径保形率可达 $96.3 \%$, 且在 $0.7 \mathrm{kPa}$ 的压力下, 流量值达 $7.36 \mathrm{~L} / \mathrm{min}$, 具有较好透气性, 实现了具有较好保形效果的泡沫铜的铣削加工过程。 关键词: 泡沫金属; 铣削加工; 孔结构; 保形率; 透气性
\end{abstract}

中图分类号: TH162

\section{Investigation of Milling Process of Foam Metal with High Porosity for Pore Structure Protection}

\author{
ZHOU Wei $^{1}$ LIU Yangxu ${ }^{1}$ CHU Xuyang ${ }^{1}$ CHEN Lu ${ }^{2}$ \\ (1. Department of Mechanical \& Electrical Engineering, Xiamen University, Xiamen 361005; \\ (2. School of Mechatronics Engineering, Harbin Institute of Technology, Harbin 150006)
}

\begin{abstract}
To protect the pore structure of foam metal in the machining process, a new milling method is proposed that different filling materials are used for solidification of pore structure. Taking copper foam as an example, the milling experiments are performed with different filling materials and processing parameters, and then surface morphology is analyzed based on the SEM results. Conformal rate of pore structure and pore crest as well as the permeability are used to evaluate the processing quality of milling process of foam metal. Using a central composite experimental design method, the quadratic regression equations are established to obtain the optimized milling parameters based on the pore size and the flow rate at fixed pressure of $0.7 \mathrm{kPa}$. The results show that better conformal effect is obtained with the hydrogenated rosin and sodium stearate at a ratio of $4: 1$. With the increase of cutting speed and feed rate, the conformal rate of pore structure and pore crest as well as the permeability are firstly increased and then decreased. Moreover, when the optimized processing parameters with a cutting speed of $311 \mathrm{r} / \mathrm{min}$ and a feed rate of $165 \mathrm{~mm} / \mathrm{min}$ are adopted, conformal rate of pore structure of $96.3 \%$, flow rate of $97.5 \%$ with the pressure of $0.7 \mathrm{kPa}$ and better permeability are obtained. In this way, the best conformal effect is obtained with optimized filling materials in the milling process of foam metal.
\end{abstract}

Key words: foam metal; milling; pore structure; conformal rate; permeability

\section{0 前言}

泡沫金属是一种在金属基体中形成无数三维孔

\footnotetext{
* 国家自然科学基金优秀青年基金(51922092)、十三五装备预研(41421020103) 和福建省杰出青年科学基金(2017J06015)资助项目。20190426 收到初稿, 20190819 收到修改稿
}

隙结构的功能材料, 分为开孔型和闭孔型两类, 前 者孔径一般为 $2 \sim 5.5 \mathrm{~mm}$, 各孔互相不连通, 后者 孔径一般为 $0.5 \sim 2.0 \mathrm{~mm}$, 各孔互相连通, 具有较高 的孔隙率，最高可达 $90 \%$ 以上 ${ }^{[1-2]}$; 泡沫金属按材质 可分为泡沫铜、泡沫镍以及泡沫铝等。不同材质泡 沫金属各具特点: 泡沫铜具有较好导电性能以及较 高导热性能, 可用于大功率组件散热以及电池负极 
材料之中; 泡沫铝具有较为突出的耐冲击耐高温的 特点, 在高层建筑业以及交通运输业应用较多; 泡 沫镍具有良好的吸音性能、电磁屏蔽性能以及透流 体性能, 可作为降噪、电波屏蔽等功能材料 ${ }^{[3-5]}$ 。鉴 于高的比表面积能够提供催化剂与气体或者液体更 多接触的机会, 泡沫金属被作为催化剂载体的首选 材料之一[6-8]。此外, 泡沫金属作为生物材料进行骨 科植入, 能够有效促进骨骼生物学固定, 体现出实 体金属不可比拟的优势 ${ }^{[9]}$ 。

目前, 对于泡沫金属的加工主要是采用特种加工 方法。SCOTT 等 ${ }^{[10]}$ 利用电火花分别加工了铁铬铝泡 沫金属、316 不锈钢泡沫金属, 研究了放电时间与进 给速度对其材料去除率的变化关系。ALEXANDER 等 ${ }^{[11]}$ 在探讨进给速度对于电火花加工泡沫金属影响 的基础上, 分析了不同密度的泡沫金属进行电火花 切割时的切除速率变化情况。MISHRA 等 ${ }^{[12]}$ 使用线 切割的方式加工多孔芯的槽结构, 利用电子显微镜 观察表面形貌并研究了线切割参数对形貌的影响。 另外, FUJIMURA 等 ${ }^{[13]}$ 利用激光对圆壳形泡沫件进 行加工, 通过小功率多次切割的方法, 实现了圆壳 形零件切割成形且没有产生表面损伤。BAUMANN 等 ${ }^{[14]}$ 提出了远程激光切割泡沫金属的工艺方法, 其 切割速度可达 $300 \mathrm{r} / \mathrm{min}$, 并对所加工的开孔金属泡 沫飞溅污垢和耐腐蚀性等进行了优化。ZHOU 等 ${ }^{[15]}$ 提出了一种基于激光加工工艺的泡沫金属快速切割 的方法, 实现泡沫金属不破坏孔隙结构的切割加工 成形。还有些学者采用传统机械加工方法对粉末烧 结的多孔金属进行加工。SCHOOP 等 ${ }^{[16]}$ 提出了一种 低温加工多孔铇的方法, 探讨了各种加工参数和冷 却条件对加工机构的影响以及切削温度的作用。LIU 等 ${ }^{[17]}$ 研究了多孔不锈钢材料微铣削过程中切削参数 对微切削性能的影响, 包括切削参数对表面形貌、 切削力、切削温度的影响。

然而, 上述加工方法在泡沫金属的加工中存在 着诸多问题。电火花加工方法虽然可以保护泡沫金 属的孔结构不在加工过程中破坏, 但是其加工效率 低、成本高, 工作液容易对孔隙结构造成污染。激 光加工能够实现泡沫金属的快速切割, 但是由于其 加工能力容易随着焦距的距离发生衰减, 加工切面 不可避免的呈雉形状, 同时, 加工热对泡沫金属具 有强氧化和腐蚀作用, 限制了泡沫金属的应用场合。 传统切削加工方法虽然可以加工粉末烧结类多孔金 属, 但是由于加工过程中的装夹力等会导致多孔金

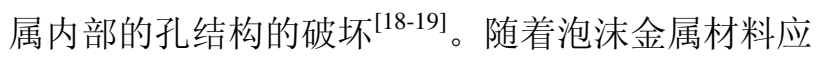
用领域的不断增多, 对泡沫金属的形状、加工精度 以及结构完整性提出了更高的要求。因此, 探索泡
沫金属的新加工方法, 避免孔隙结构的变形的研究 工作具有十分重要的意义。

本文提出了利用填充材料进行泡沫金属固化后 再进行保形铣削加工的新方法, 开展不同类型填充 材料的铣削加工试验, 借助电镜观察, 孔径统计分 析、通气性试验测试等方法确定了具有最佳保形效 果的填充材料类型以及探讨了铣削加工参数对表面 质量的影响。通过中心组合试验建立了孔结构尺寸、 透气性与铣削加工参数之间的二次回归方程, 获得 优化的铣削加工参数。

\section{1 试验}

\section{1 填充材料选择}

填充材料对保形加工的质量具有至关重要的影 响, 需要具有如下三个特点。

（1）填充材料具有一定的刚度和韧性。填充材 料在常温条件下应具有一定的强度和硬度, 不会轻 易被切削力和装夹力压溃、崩裂。此外, 加工过程 中由于多刀铣刀对工件的间断冲击，泡沫金属的刚 度分布不均匀导致切削力不断变化, 填充材料还必 须具有一定的韧性。

(2) 填充材料具有合适的相变温度。为方便填 充材料融化后与泡沫金属分离, 填充材料相变温度 不宜过高。但相变温度太低, 会导致加工过程中产 生的切削热融化填充材料, 使其丧失保形能力。此 外, 液态下的填充材料黏度要较小, 有利于在泡沫 金属的均匀渗透, 填充充分。

（3）考虑到安全、污染等因素，填充材料应具有 不易燃易爆，不挥发升华，无毒无害的等特点。综合 填充材料选择标准, 选择如下几种可行的材料对泡沫 金属进行填充并后续进行铣削试验材料, 其相变特 点、填充渗透现象以及对应清洗剂, 如表 1 所示。

图 1 为泡沫金属的保形填充与清洗铣削加工过 程示意图。首先, 将填充物放入加热模具中, 在电 热板上进行加热, 模具中铺上锡管纸以利于填充材 料、填充废料的取出。然后，将泡沫金属放入熔融 的液态填充材料中, 在毛细效应与重力的作用下, 熔融的填充材料逐渐向多孔金属内部渗透。而后, 保持加热一段时间并不断翻转被填充材料直至气泡 完全排除。最后, 将填充完的泡沫金属取出, 等待 泡沫金属内部填充液冷却凝固，完成填充。对于铣 削后的泡沫金属, 需对填充材料进行清洗。在熔融 的填充材料下, 根据填充材料溶解性等物理性质, 选择相应的溶解液进行溶解, 并用超声波振动装置 进行辅助清洗。 
表 1 不同材料在泡沫金属内部的填充特性

\begin{tabular}{|c|c|c|c|c|c|}
\hline 序号 & 材料 & 加热温度 ${ }^{\circ} \mathrm{C}$ & 渗透速度 & 特点 & 溶解剂 \\
\hline 1 & 石蜡 & 150 & 快 & $\begin{array}{c}\text { 融化迅速, 呈无色透明状液体, 黏度较小。凝固后填充均匀 } \\
\text { 质软, 㓞性高 }\end{array}$ & 煤油 \\
\hline 2 & 氢化松香 & 300 & 较快 & $\begin{array}{c}\text { 可完全融化, 呈淡黄色液体, 具有一定黏性 } \\
\text { 凝固后, 硬度高, 脆性略低 }\end{array}$ & 乙醇 \\
\hline 3 & 松香 & 250 & 较快 & 可完全融化成淡黄色液体, 黏度较小。凝固后较脆, 硬度高 & 乙醇 \\
\hline 4 & $\begin{array}{l}\text { 松香与硬脂酸 } \\
\text { 钠混合(3:1) }\end{array}$ & & & & \\
\hline 5 & $\begin{array}{l}\text { 松香与硬脂酸 } \\
\text { 钠混合 }(4: 1)\end{array}$ & $\begin{array}{l}\text { 随着硬脂酸钠的增 } \\
\text { 加, 渗透速度减慢 }\end{array}$ & & $\begin{array}{l}\text { 松香很快融化, 硬脂酸钠随后形成白色絮状物, 在松香的溶解下, 完全 } \\
\text { 混合, 黍度随硬脂酸钠的增加而增加。凝固后具有较高的硬度与韧性 }\end{array}$ & 乙醇 \\
\hline 6 & $\begin{array}{l}\text { 松香与硬脂酸 } \\
\text { 钠混合(5:1) }\end{array}$ & & & & \\
\hline
\end{tabular}

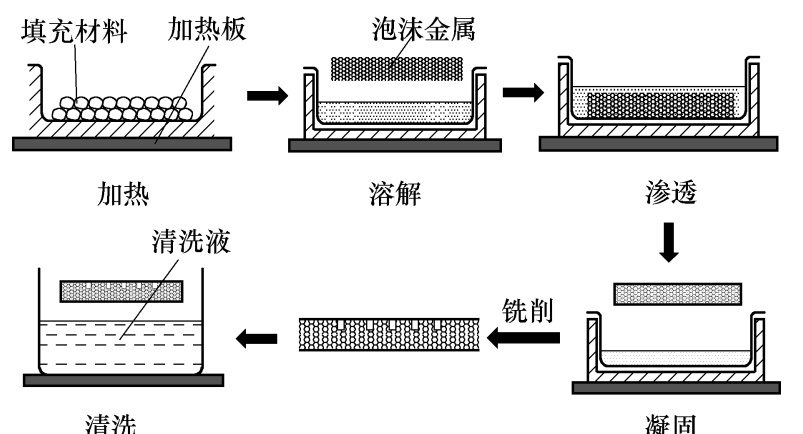

图 1 泡沫金属的保形铣削加工过程示意图

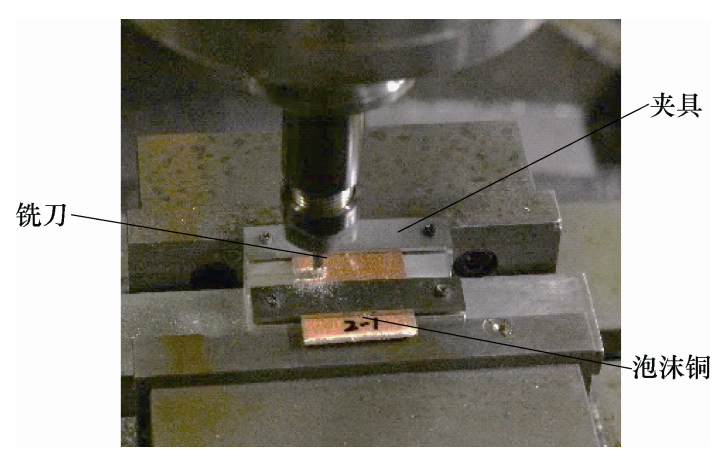

图 2 泡沫金属铣削加工的现场图

\section{2 泡沫金属的保形铣削加工试验设计}

本试验研究对象尺寸为 $70 \mathrm{~mm} \times 40 \mathrm{~mm} \times 2 \mathrm{~mm}$ 的泡沫铜 ${ }^{[20]}$, 考虑到其厚度较薄, 可实现一次加工 成形。因此, 将背吃刀量设置为 $2 \mathrm{~mm}$, 进而主要研 究填充材料、主轴转速、进给速度对保形质量的影 响。首先设定主轴转速为 $300 \mathrm{r} / \mathrm{min}$, 进给速度为 160 $\mathrm{mm} / \mathrm{min}$, 分别通过对 6 种不同填充材料填充的泡沫 金属进行铣削加工, 通过 SEM 观察表面质量, 初步 确定保形效果较好的填充材料。然后进行改变主轴 转速、进给速度的单因素试验, 并基于切割面表面 统计、透气性测试两种定量指标, 进行保形效果测 试, 进一步研究填充材料、铣削加工参数对泡沫金 属保形加工质量的影响关系, 从而确定最优保形填 充材料。最后以进行正交试验, 建立二次回归方程, 寻求最优保形铣削参数。单因素试验具体参数设置 如下: 当主轴转速为 $300 \mathrm{r} / \mathrm{min}$ 时进给速度分设置为 $120 \mathrm{~mm} / \mathrm{min}, 140 \mathrm{~mm} / \mathrm{min}, 160 \mathrm{~mm} / \mathrm{min}, 180$ $\mathrm{mm} / \mathrm{min}, 200 \mathrm{~mm} / \mathrm{min}$; 当进给速度为 $160 \mathrm{~mm} / \mathrm{min}$ 时, 主轴转速分别为 $100 \mathrm{r} / \mathrm{min}, 200 \mathrm{r} / \mathrm{min}, 300 \mathrm{r} / \mathrm{min}$, $400 \mathrm{r} / \mathrm{min}, 500 \mathrm{r} / \mathrm{min}$, 该加工试验现场如图 2 所示。

\section{3 泡沫金属的保形加工质量分析}

\subsection{1 孔径统计测试}

泡沫金属的孔结构是诸多优良特性的物理基 础 ${ }^{[21]}$, 因此在加工过程应该尽量避免孔结构破坏。
加工前后铣削表面孔径大小改变程度、嵴的塑性形 变程度可作为孔结构完整性的重要指标。基于 Nano measurer 软件对切削截面 SEM 图片进行孔径直径 与孔嵴的尺寸统计, 每组加工表面选择 7 组不同位 置进行测量, 共包括 105 个孔径尺寸以及 175 个崉 尺寸。统计前设定标尺, 然后通过划定孔径与孔嵴 尺寸获取统计结果。

由文献[22]可知，激光加工的泡沫金属孔结构 变形很小, 具有很好的保形效果。因此, 设定激光 加工的泡沫金属表面作为标准，与铣削保形加工的 表面作对比，并引入保形率概念进行保形铣削效果 的定量表征。由于保形铣削加工后, 保形效果好的 泡沫金属, 不容易出现孔径堵塞、孔嵴压溃等情况。 因此, 孔径、孔嵴平均尺寸越接近于激光切割表面 质量，保形效果越佳。

对于嵴保形率，由于铣削保形加工后嵴平均尺 寸增大, 设激光加工后表面嵴平均尺寸为 $B_{1}$, 保形 铣削加工后统计的嵴平均尺寸为 $B_{0}$, 则崉保形率 $y_{0}$ 可表示为

$$
y_{0}=\frac{B_{1}}{B_{0}} \times 100 \%
$$

对于孔径保形率, 由于保形铣削加工后孔结构 平均尺寸减小, 设激光加工后表面孔结构的平均尺 寸为 $A_{1}$, 铣削加工后统计的切割面孔结构的平均尺 
寸为 $A_{0}$ 。则孔径保形率 $y_{1}$ 表示为

$$
y_{1}=\frac{A_{1}}{A_{0}} \times 100 \%
$$

\subsection{2 透气性测试}

泡沫金属在换热传热、过滤、催化剂载体等 方面的应用, 是依靠其内部流体在多孔结构中的 流动与传输 ${ }^{[23]}$, 因此需要对加工后的多孔金属进 行透气性测试。对于泡沫金属的透气性能研究方 面, 普遍认为较低流速的流体处于黏性流动状态 而满足达西定律 ${ }^{[24-25]}$

$$
\frac{\Delta p}{L}=\frac{\mu}{K} \frac{Q}{A}
$$

式中, $\Delta p$ 为透过泡沫金属的压降值; $L$ 为沿流体流 动方向泡沫金属的长度; $\mu$ 为流体黏度系数; $K$ 为 渗透因子; $A$ 为流体通过泡沫金属的截面面积; $Q$ 为流量。

试验使用材料为保形铣削后的泡沫铜, 其尺寸为 $70 \mathrm{~mm} \times 12 \mathrm{~mm} \times 2 \mathrm{~mm}$, 且两侧均为切割表面, 将其 3 片按串联方式放入测试装置中进行透气性测试。测试 系统示意图, 如图 3 所示。该系统包括测试装置、空 气流量计、压力传感器等。气体从反应器入口进入泡 沫铜载体中, 通过泡沫铜到达到出气口, 然后沿出气 导管直接进入大气环境。在与进气口相对的地方, 设 有压力传感器, 且出口管与外界大气相通, 因此数字 压力计的数值即可表示进气口压力值与出气口压力 的差值。通过不同的进气流速变化, 可反应各加工参 数下透过泡沫铜的气体压力-流速关系。

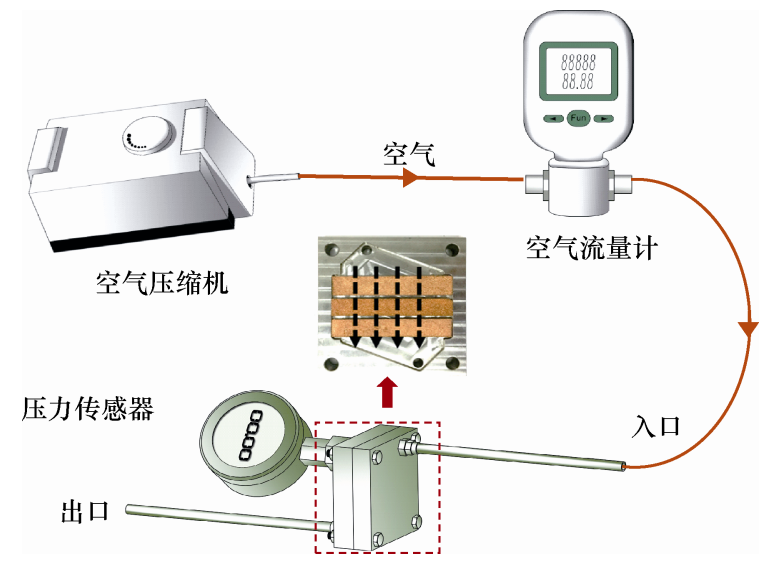

图 3 泡沫金属的透气性测试系统示意图

测试前, 进行装置密封性测试, 封闭装置出气端。 使用微量注射洜对注射剂施加一定的位移, 使装置内 部整体气压升高, 若压力计示数稳定不变, 则说明装 置气密性良好。试验测试阶段, 调节空压机功率使得 进气端流体流速不断变化, 通过流量计记录气体流 速, 通过压力传感器记录相对应的稳定的压力值。

\section{4 基于中心组合设计的泡沫金属保形铣削参数 优化}

选用具有最佳的填充材料的泡沫铜作为研究 对象, 将主轴转速, 进给速度作为自变量, 以孔 径尺寸、透气性测试中的流量值作为目标量, 通 过中心组合设计试验方案建立回归方程, 求取切 削参数最优值。中心组合设计示意图如图 4 所示, 共进行 9 次试验, 其具体分为三类, 即轴向点 水平试验、星 $\star$ 点试验以及中心点 $\mathrm{O}$ 零水平试验。 试验的目标量用 $y$ 表示, 中心组合设计试验参数, 如表 2 所示。

B

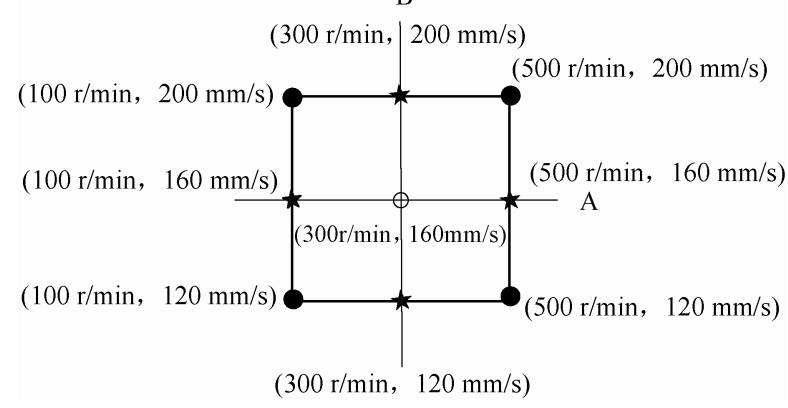

图 4 中心组合设计试验

假设有 $m$ 个试验因素即自变量 $x_{j}, j=1,2, \cdots, m$, 则二次回归方程的一般形式为

$$
\begin{gathered}
\hat{y}=b_{0}+\sum_{j=1}^{m} b_{j} x_{j}+\sum_{k<j} b_{k j} x_{k} x_{j}+\sum_{j=1}^{m} b_{j j} x_{j}^{2} \\
k=1,2, \cdots, m-1(j \neq k) \\
b_{0}=\frac{1}{n} \sum_{i=1}^{n} y_{i}=\bar{y} \\
b_{j}=\frac{\sum_{i=1}^{n} z_{j i} y_{i}}{\sum_{i=1}^{n} z_{j i}{ }^{2}} \quad j=1,2, \cdots, m \\
\left.b_{k j}=\frac{\sum_{i=1}^{n}\left(z_{k} z_{j}\right)_{i} y_{i}}{\sum_{i=1}^{n}\left(z_{k} z_{j}\right)_{i}{ }^{2} \quad} j^{j}>k, k=1,2, \cdots, m-1\right) \\
b_{j j}=\frac{\sum_{i=1}^{n}\left(z_{j i}^{\prime}\right) y_{i}}{\sum_{i=1}^{n}\left(z_{j i}^{\prime}\right)^{2}} \\
\sum_{i=1}^{m} z_{i}=\frac{x_{i}-\left(x_{m}+x_{1}\right) / 2}{\left(x_{m}-x_{1}\right) / m} \\
z_{i}{ }^{2}-\frac{1}{n} \sum_{i=1}^{m} \sum_{j=1}^{n} z_{i j}{ }^{2}
\end{gathered}
$$


式中, $n$ 为总共的试验次数; $\bar{y}$ 为 9 次试验结果的 平均值。在本次试验中, 试验因素 $m$ 值为 2 , 对建 立的回归方程进行显著性检验。

$$
\begin{gathered}
S S_{T}=\sum_{i=1}^{n} y_{i}{ }^{2}-\frac{1}{n}\left(\sum_{i=1}^{n} y_{i}\right)^{2} \\
S S_{R}=\sum S S_{1}+\sum S S_{2}+\sum S S_{3} \\
S S_{e}=S S_{T}-S S_{R} \\
S S_{j}=b_{j}{ }^{2} \sum_{i=1}^{n} z_{j i}{ }^{2}, j=1,2, \cdots, m \\
S S_{k j}=b_{k j}{ }^{2} \sum_{i=1}^{n}\left(z_{k} z_{j}\right)_{i}{ }^{2} j>k, k=1,2, \cdots, m-1 \\
S S_{j j}=b_{j j}{ }^{2} \sum_{i=1}^{n}\left(z_{j i}\right)^{2}
\end{gathered}
$$

对回归方程的自变量进行偏导数求解, 则可得 到回归方程极值以及所对应自变量数值, 该值则为 最优化的加工参数。

\section{表 2 保形铣削加工的试验参数}

\begin{tabular}{cccc}
\hline 符号 & 因素 & 水平 1 & 水平 2 \\
\hline A & 主轴转速 $/(\mathrm{r} / \mathrm{min})$ & 100 & 500 \\
$\mathrm{~B}$ & 进给速度 $/(\mathrm{mm} / \mathrm{s})$ & 120 & 200 \\
\hline
\end{tabular}

\section{2 结果与讨论}

\section{1 填充材料对铣削表面形貌的影响}

图 5 是在 6 种不同材料填充下的泡沫金属经过铣 削加工后的 SEM 图。铣削加工参数为: 主轴转速为 $300 \mathrm{r} / \mathrm{min}$, 进给速度为 $160 \mathrm{~mm} / \mathrm{s}$ 。石蜡、松香、松香 与硬脂酸钠质量比为 $5: 1$ 混合材料的保形效果明显较 差(图 5f), 这可能是由于石蜡硬度过小, 受切削力的 作用下容易引起孔结构的变形, 而松香较脆, 刚度差, 切削过程中容易碎成粉末以致与泡沫金属的壁面接 触不紧密。而氢化松香与硬脂酸纳质量比为 5:1 的混 合填充材料, 由于硬脂酸纳含量少, 对填充后的泡沫 金属的刚度、㓞性的不高, 保形铣削加工性能提升不 明显。当选用氢化松香、松香与硬脂酸钠质量比为 3:1 混合材料、松香与硬脂酸钠质量比为 $4: 1$ 混合材 料, 通过电镜表面观察的孔隙结构基本一致。

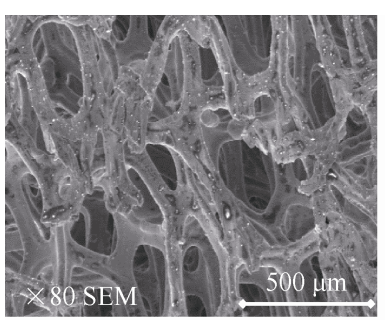

(a) 石蜡

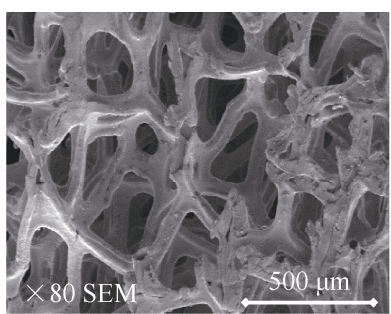

(b) 松香

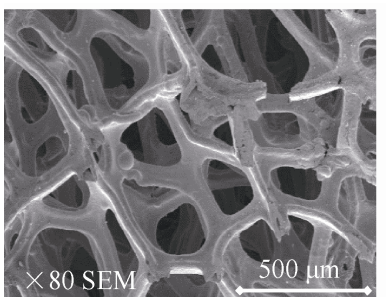

(c) 氢化松香

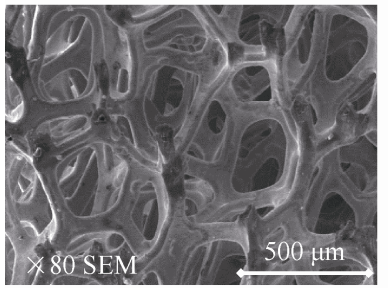

(e) 松香硬脂酸钠 $4: 1$

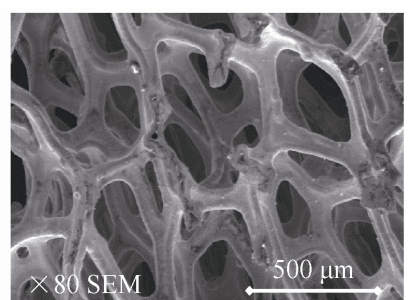

(d) 松香硬脂酸钠 $3: 1$

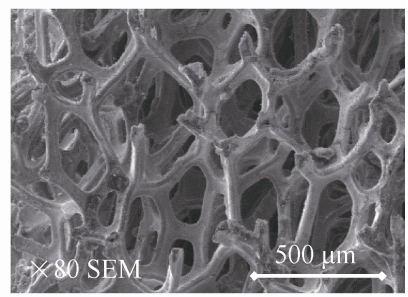

(f) 松香硬脂酸钠 $5: 1$
图 5 不同材料填充保形铣削表面形貌对比

图 6 是在选用氢化松香、松香与硬脂酸钠 3:1(质量 比)混合、松香与硬脂酸钠 4:1(质量比)混合 3 种材料填 充下的泡沫金属的孔结构统计测试结果与透气性测试 结果。由图可知, 对松香与硬脂酸钠 4:1(质量比)混合 填充的泡沫金属进行保形铣削加工, 其孔嵴保形率、 孔径保形率可达: 91.5\%, 96.7\%, 分别高于松香的 9.8\%, 14.2\%、高于松香与硬脂酸纳 3:1(质量比)混合的 2.1\%, $4.3 \%$ 。其可能原因在于, 硬脂酸钠具有的优良可加工 性能大大增加了其填充材料的刚度、韧性, 结合松香 不易变形的高硬度特性, 其保形效果远优于氢化松香。

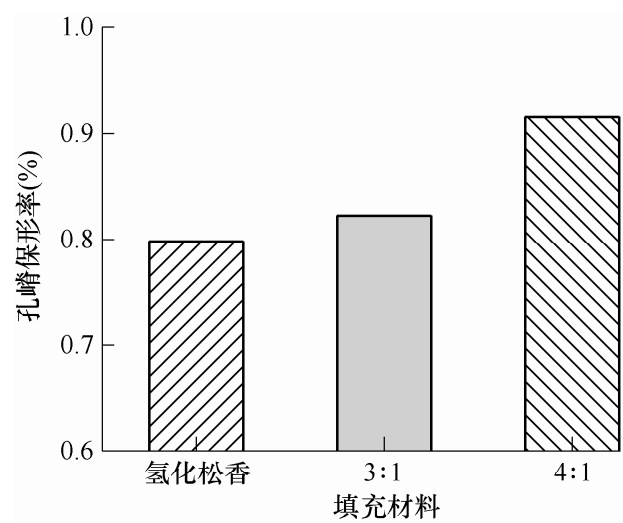

(a) 孔崉保形率

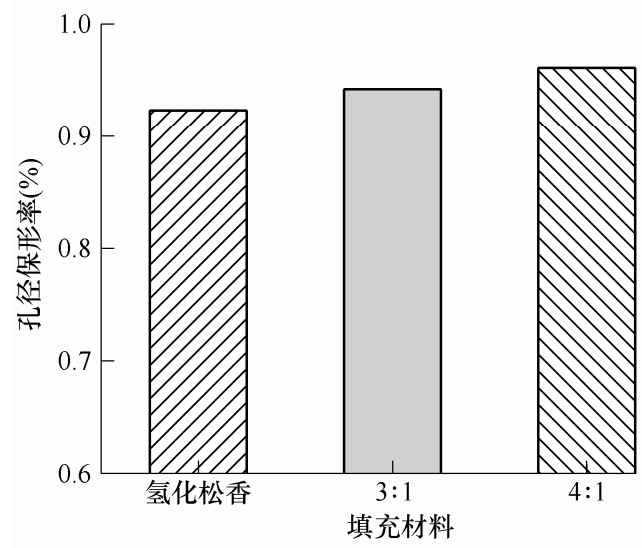

(b) 孔径保形率 


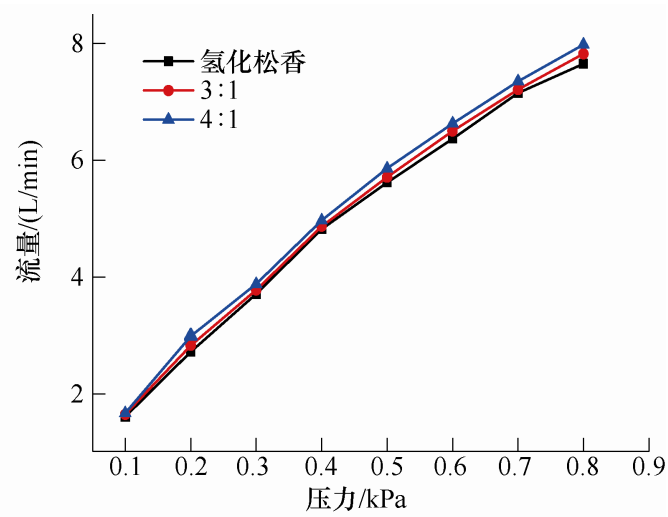

(c) 压力-流速变化关系

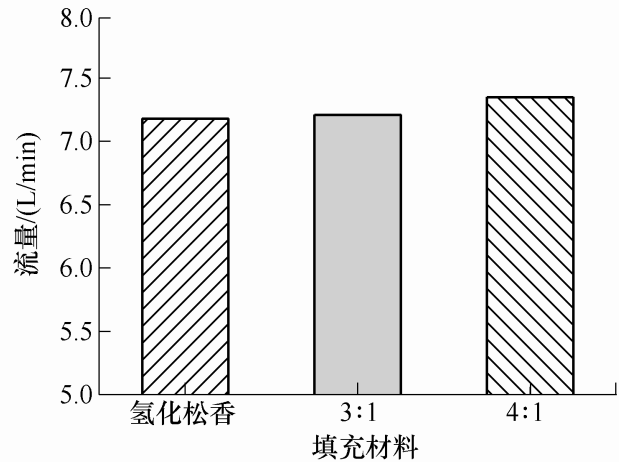

(d) 不同填充材料 $0.7 \mathrm{kPa}$ 的流量变化

图 6 不同材料填充保形铣削表面孔径统计与通气性测试

此外, 对于松香与硬质酸纳 3:1(质量比)而言, 随着 硬脂酸钠的增加, 材料黏度增加, 这导致在加工过 程中发生黏刀的现象使得加工质量下降。对于松香 与硬脂酸纳 5:1(质量比)随着而言, 随着硬脂酸钠的 降低, 又会出现可加工性降低, 㓞性减小从而导致 加工质量下降。因此, 当松香与硬脂酸钠按 4:1(质 量比)混合填充的泡沫金属在保形铣削加工中表现 出最佳的保形效果。

\section{2 加工参数对铣削表面形貌的影响}

\subsection{1 进给速度的影响}

图 7 是在不同进给速度下孔结构保形率、孔嵴 保形率以及透气性变化图。从该图可以看出孔结构保 形率、孔嵴保形率以及透气性能均随着进给速度的增 加呈现先增大后减小的趋势, 且在进给速度为 160 $\mathrm{mm} / \mathrm{min}$ 具有最高保形率。孔结构保形率、孔嵴保形 率分别可达 92.3\%, 97.5\%。在切割的过程中, 孔径被 破坏主要是由于孔隙四周的金属层在切削力作用下 发生推移, 从而搬运到孔隙之中。当进给速度较小时, 刀具在加工表面的停留时间增加, 较长停留时间导致 铣削表面孔结构沿铣削方向变形加剧 ${ }^{[26]}$, 加工质量效 果降低。随着进给速度的增加, 停留时间降低, 表层 金属塑变现象减少, 切割面质量变好, 因此孔结构与 崉的保形率均大幅上升; 当进给速度进一步增大, 泡 沫金属切割过程在进给方向以挤压破坏为主 ${ }^{[27]}$, 使得
此推移过程变得剧烈, 更多的金属层被移至孔隙当 中，孔隙与嵴的保形率较小，保形效果变差。

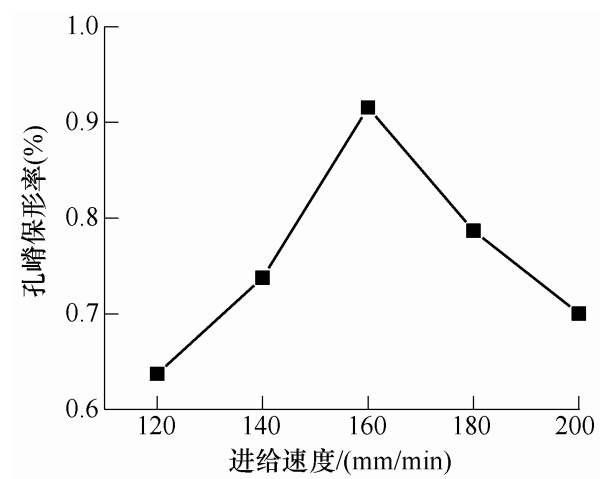

(a) 孔崉保形率变化曲线

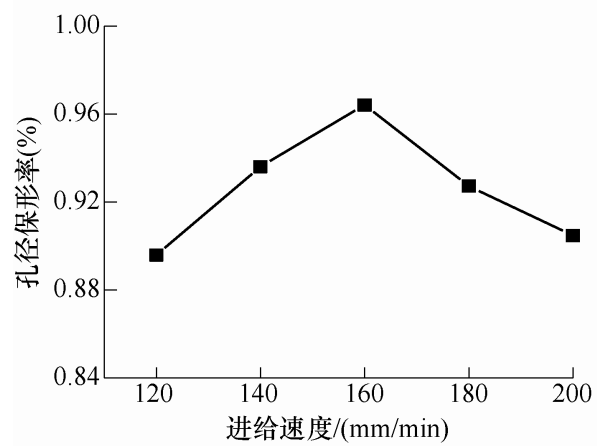

(b) 孔径保形率变化曲线

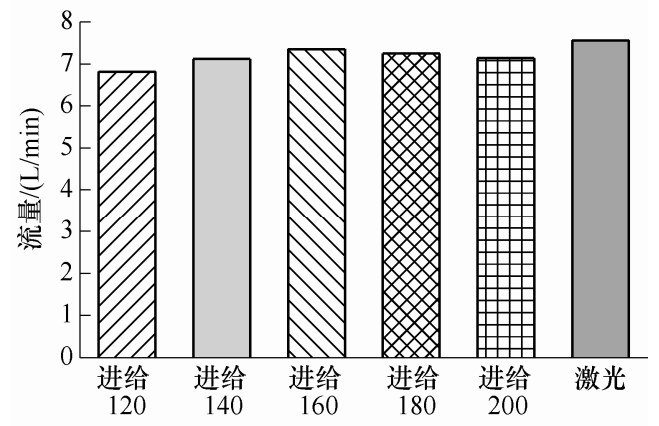

(c) $0.7 \mathrm{kPa}$ 时流量变化关系

图 7 在不同进给速度下孔结构保形率、孔崉保形率 以及透气性变化图(主轴转速为 $300 \mathrm{r} / \mathrm{min}$ )

\subsection{2 刀具转速的影响}

图 8 是不同刀具转速下孔径保形率、孔嵴保形 率以及透气性变化图。从该图可以看出, 孔径保形 率、孔嵴保形率以及透气性能均随着进给速度的增 加呈现先增大后减小的趋势, 且在进给速度为 300 $\mathrm{r} / \mathrm{min}$ 时具有最高保形率。当主轴转速较小时, 切削 力大, 刀刃在加工表面停留时间较长, 对于孔结构 破坏更为严重; 随着转速适当提高, 单位时间切割 材料减少, 切割能力增强, 切割过程以剪切断裂变 形为主, 切割面孔结构完整度增加, 孔径与㗳保形 率明显上升; 当转速进一步增大时, 由断续切割理 论可知 ${ }^{[28]}$, 过高的转速导致切削力变化频繁, 工件 振动加剧, 表面摩擦加剧, 表面塑性形变和结构弯 
曲程度更加明显, 保形效果降低。

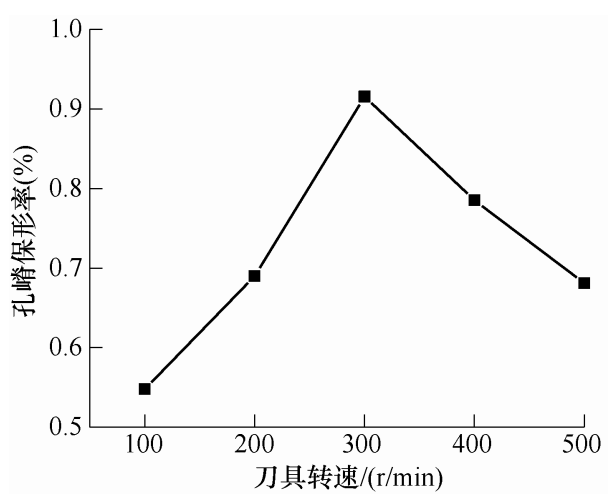

(a) 孔崉保形率变化曲线

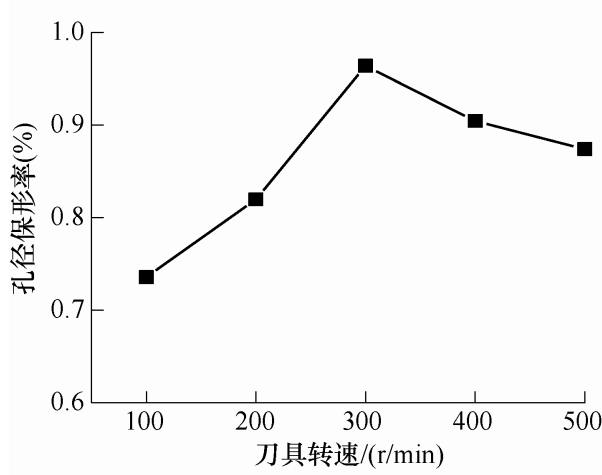

(b) 孔径保形率变化曲线

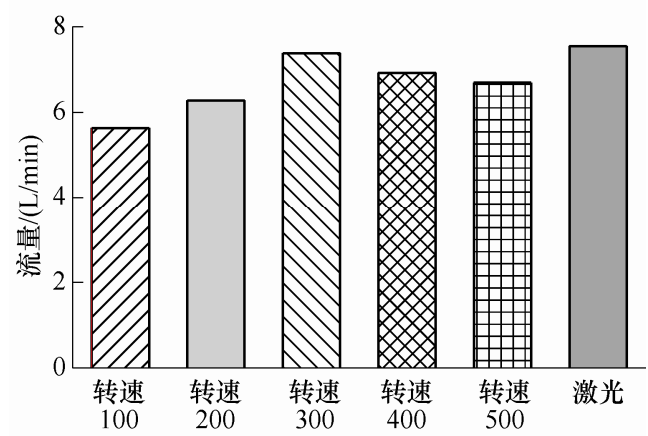

(c) $0.7 \mathrm{kPa}$ 时流量变化关系

图 8 不同转速下孔隙保形率、孔嵴保形率以及 透气性变化图(进给速度为 $160 \mathrm{~mm} / \mathrm{min}$ )

\section{3 加工参数优化}

根据中心组合试验, 不同因素下孔径统计的孔 径与透气性试验中的 $0.7 \mathrm{kPa}$ 所对应的流量值分别由 $y_{1}, y_{2}$ 表示, 如表 3 所示, 联立方程式(4) (10)可以 得到孔径与刀具转速与进给速度之间的关系式

$$
\begin{gathered}
y_{1}=-0.4109+0.0011 x_{1}+0.0088 x_{2}+8 \times 10^{-7} x_{1} x_{2}- \\
2.0325 \times 10^{-6} x_{1}^{2}-2.7437 \times 10^{-5} x_{2}^{2}
\end{gathered}
$$

同理可得流量与转速和进给速度之间的关系式 $y_{2}=1.3972+0.0071 x_{1}+0.0583 x_{2}+6.875 \times 10^{-6} x_{1} x_{2}-$

$$
1.2925 \times 10^{-5} x_{1}^{2}-1.825 \times 10^{-4} x_{2}^{2}
$$

联立方程(11) (15)对回归方程式(17)、(18)进
行方差分析, 并根据式(9)、(10)对刀具转速 $\mathrm{x}_{1}$, 进 给速度 $x_{2}$ 进行中心化处理, 获得参数 $\mathrm{z}_{1}, \mathrm{z}_{2}, \mathrm{z}_{1}^{\prime}, \mathrm{z}_{1}^{\prime}$ 。 其中变形后的方程为

$$
\begin{gathered}
y_{1}=0.3981+0.02 z_{1}+0.0086 z_{2}+0.0064 z_{1} z_{2}- \\
0.0813 z_{1}^{\prime}-0.0439 z_{2}^{\prime} \\
y_{2}=7.2960+0.8465 z_{1}+1.465 z_{2}+0.055 z_{1} z_{2}- \\
0.517 z_{1}^{\prime}-0.292 z_{2}^{\prime}
\end{gathered}
$$

表 3 中心组合试验结果

\begin{tabular}{ccccccc}
\hline$z_{1}$ & $z_{2}$ & $z_{1} z_{2}$ & $z_{1}^{\prime}$ & $z_{2}^{\prime}$ & $y_{1}$ & $y_{2}$ \\
\hline 1 & 1 & 1 & $1 / 3$ & $1 / 3$ & 0.387 & 6.95 \\
1 & -1 & -1 & $1 / 3$ & $1 / 3$ & 0.350 & 6.63 \\
-1 & 1 & -1 & $1 / 3$ & $1 / 3$ & 0.348 & 6.75 \\
-1 & -1 & 1 & $1 / 3$ & $1 / 3$ & 0.337 & 6.65 \\
1 & 0 & 0 & $1 / 3$ & $-2 / 3$ & 0.435 & 7.27 \\
-1 & 0 & 0 & $1 / 3$ & $-2 / 3$ & 0.366 & 6.91 \\
0 & 1 & 0 & $-2 / 3$ & $1 / 3$ & 0.441 & 7.19 \\
0 & -1 & 0 & $-2 / 3$ & $1 / 3$ & 0.436 & 7.14 \\
0 & 0 & 0 & $-2 / 3$ & $-2 / 3$ & 0.480 & 7.35 \\
\hline
\end{tabular}

方程分析结果如表 4、5 所示。可以看到, 两回 表 4 孔径回归方程方差分析结果

\begin{tabular}{cccccc}
\hline 差异量 & $S S$ & $d f$ & $M S$ & $F$ & 显著性 \\
\hline $\mathrm{z}_{1}$ & 0.002405 & 1 & 0.002405 & 3.297 & \\
$\mathrm{z}_{2}$ & 0.0004402 & 1 & 0.000440 & 2.498 & \\
$\mathrm{z}_{1} \mathrm{z}_{2}$ & 0.0001652 & 1 & 0.0001652 & 0.821 & \\
$\mathrm{z}_{1}{ }^{\prime}$ & 0.01321 & 1 & 0.01321 & 36.227 & $* *$ \\
$\mathrm{z}_{2}{ }^{\prime}$ & 0.003844 & 1 & 0.003844 & 11.545 & $*$ \\
回归分析 & 0.80154 & 5 & 0.1603 & 10.877 & $*$ \\
残差 & 0.04421 & 3 & 0.01473 & & \\
总计 & 0.8457 & 8 & & & \\
\hline
\end{tabular}

表 5 流量回归方程方差分析结果

\begin{tabular}{cccccc}
\hline 差异量 & $S S$ & $d f$ & $M S$ & $F$ & 显著性 \\
\hline $\mathrm{z}_{1}$ & 0.002405 & 1 & 0.002405 & 3.297 & \\
& 0.000440 & 1 & 0.0004402 & 2.498 & \\
$\mathrm{z}_{2}$ & 2 & & & & \\
& 0.000165 & 1 & 0.0001652 & 0.821 & \\
$\mathrm{z}_{1} \mathrm{z}_{2}$ & 2 & & & & \\
$\mathrm{z}_{1}{ }^{\prime}$ & 0.01321 & 1 & 0.01321 & 36.227 & $* *$ \\
$\mathrm{z}_{2}{ }^{\prime}$ & 0.003844 & 1 & 0.003844 & 11.545 & $*$ \\
回归分析 & 0.8015 & 5 & 0.1603 & 10.877 & $*$ \\
残差 & 0.04421 & 3 & 0.01473 & & \\
总计 & 0.8457 & 8 & & & \\
\hline
\end{tabular}

归方程的系数显著水平相似, 当 $F>F_{0.05}(1,3)=10.13$ 时, 为显著; 当 $F>F_{0.01}(1,3)=34.12$ 时, 为非常显著, 
因此转速的平方对孔直径、流量水平的有非常显著 $(* *)$ 的影响, 进给速度的平方对孔直径、流量水平 有显著 $(*)$ 的影响。

根据线性回归方程, 利用 Matlab 软件对上述孔 隙直径和流量值两回归方程的三维曲面图如图 9 所

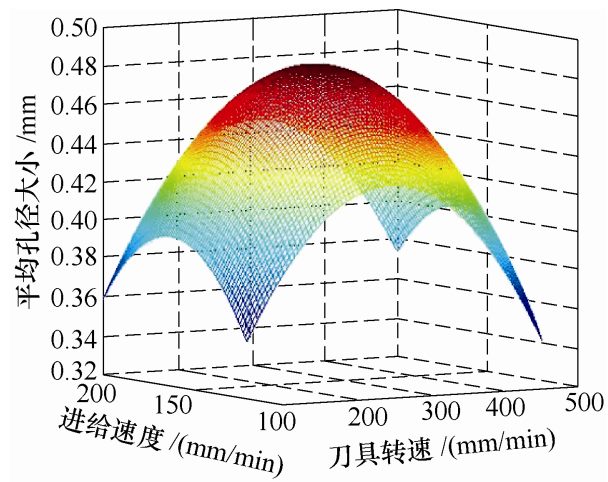

(a) 孔隙尺寸三维图

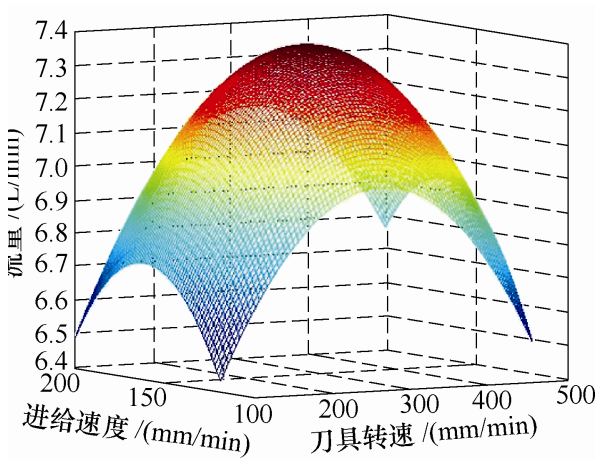

(b) 流量值三维图

图 9 二次回归方程的三维曲面图

示。由曲面图可以发现, 其变化趋势与上文在选定 转速和选定进给速度时孔径与流量值变化趋势相一 致, 且在转速为 $300 \mathrm{r} / \mathrm{min}$, 进给速度为 $160 \mathrm{~mm} / \mathrm{min}$ 左右有最大值。令 $\frac{\partial y}{\partial x_{1}}=0, \frac{\partial y}{\partial x_{2}}=0$, 则可得到孔径 尺寸和流量值两个回归方程的极值。求解可知, 当 刀具转速 $x_{1}=303.2 \mathrm{r} / \mathrm{min}$, 进给速度 $x_{2}$ 为 164.8 $\mathrm{mm} / \mathrm{min}$ 时, 孔径尺寸有最大值为 $0.4808 \mathrm{~mm}$; 当 刀具转速 $x_{1}=318.7 \mathrm{r} / \mathrm{min}$, 进给速度 $x_{2}$ 为 165.7 $\mathrm{mm} / \mathrm{min}$ 时, 定压强下流量值有最大值为 7.3597 $\mathrm{L} / \mathrm{min}$; 综合考虑两个参数对加工表面质量的影响, 选定刀具转速为 $311 \mathrm{r} / \mathrm{min}$, 进给速度为 $165 \mathrm{~mm} / \mathrm{min}$ 进行验证试验。

\section{4 优化加工参数的验证}

使用氢化松香-硬脂酸钠 (4:1)材料填充泡沫金 属, 在转速为 $311 \mathrm{r} / \mathrm{min}$, 进给速度为 $165 \mathrm{~mm} / \mathrm{min}$ 的 加工参数下进行铣削加工参数的验证试验。对铣削 加工后的泡沫金属表面的表面形貌、孔结构尺寸统 计和透气性测试进行分析。图 10 为优化加工参数下 铣削加工表面与激光加工表面形貌对比。从该图可
以看出, 使用氢化松香-硬脂酸钠(4:1)材料填充, 在 优化参数条件下铣削加工后的泡沫金属表面质量与 激光切割的表面质量相似, 孔结构完整, 无坍塌堵 塞等现象出现。通过孔结构的尺寸统计得出, 孔径 平均尺寸为 $0.4805 \mathrm{~mm}$, 嵴平均尺寸为 $0.0891 \mathrm{~mm}$, 保形率分别为 $96.3 \%$ 和 $93.7 \%$ 。透气性测试得压强 在 $0.7 \mathrm{kPa}$ 时, 通过保形铣削加工的泡沫金属表面的 流量值为 $7.36 \mathrm{~L} / \mathrm{min}$, 为同条件下通过激光加工表 面流量值的 $97.5 \%$ 。因此, 使用氢化松香-硬脂酸钠 (4:1)材料填充并在优化加工参数条件下进行的泡沫 金属铣削加工具有较好的保形效果。

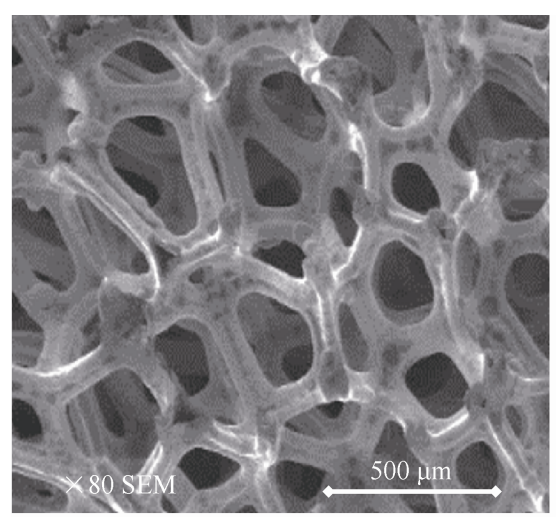

(a) 铣削加工表面

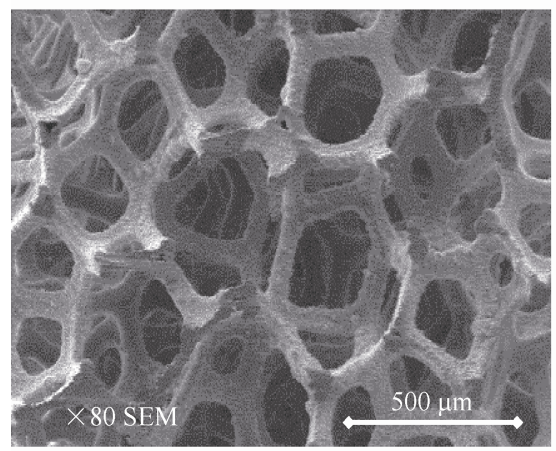

(b) 激光加工表面

图 10 优化加工参数下铣削加工表面与激光 加工表面形貌对比

\section{3 结论}

为保护泡沫金属的孔结构在加工过程中不被破 坏, 本文以泡沫铜为加工材料, 对其进行了不同材料 的填充以及固化过程对比试验, 在不同铣削加工参数 条件下进行了泡沫铜的铣削加工试验, 通过表面形貌 观察, 孔结构尺寸统计和透气性测试评价了在不同参 数下铣削加工的保形效果。利用中心组合设计试验, 建立了评价指标的回归方程获得优化的加工参数, 并 对选用优化的填充材料、优化的铣削加工参数的泡沫 金属进行加工试验, 主要得到以下结论。 
(1) 对不同填充材料、主轴转速以及进给速度 进行了铣削加工试验, 借助 SEM 表面形貌定量观 测, 孔结构尺寸统计和透气性测试评价其保形质量, 研究结果表明以氢化松香-硬脂酸钠(质量比为 4:1) 为填充材料, 其保形效果最好; 同时保形率与透气 性随刀具转速、进给速度的增加呈现出先增大后减 小的趋势, 其中转速对表面质量的影响要比进给速 度对表面质量的影响更显著。在转速为 $300 \mathrm{r} / \mathrm{min}$, 进给速度为 $160 \mathrm{~mm} / \mathrm{min}$ 时, 表现出最好的保形效果。

（2）选用优化的填充材料开展泡沫金属的保形 铣削加工的中心组合试验, 根据测试结果建立二次 回归方程, 得到转速 $311 \mathrm{r} / \mathrm{min}$, 进给速度 165 $\mathrm{mm} / \mathrm{min}$ 为优化的铣削加工参数。使用优化的加工 参数对泡沫铜进行保形铣削加工的验证试验, 其结 果为: 孔径保形率为 $96.4 \%$, 崉保形率为 $93.7 \%$, 流量值为 $7.36 \mathrm{~L} / \mathrm{min}$, 跟激光加工表面流量值相差 不大。因此, 选用优化填充材料, 并以优化加工参 数进行泡沫金属铣削加工可以获得较好的孔结构完 整性与透气性, 表明该种加工方法可以实现较好的 孔结构保形效果。

\section{参 考 文 献}

[1] WAMG M, PAN Ning. Modeling and prediction of the effective thermal conductivity of random open-cell porous foams[J]. International Journal of Heat and Mass Transfer 2008, 51: 1325-1331.

[2] SONG Z, KISHIMOTO S. The cell size effect of closed cellular materials fabricated by pulse current assisted hot isostatic pressing on the compressive behavior[J]. Scripta Materialia, 2006, 54(8): 1531-1535.

[3] 张勇, 林福泳. 铝泡沫填充薄壁结构耐撞可靠性优化设 计[J]. 机械工程学报, 2011, 47(22): 93-99.

ZHANG Yong, LIN Fuyong. Crashworthiness reliability design optimization of aluminum foam filled thin-wall structures[J]. Journal of Mechanical Engineering, 2011, 47(22): 93-99.

[4] 池勇, 汤勇, 陈锦昌, 等. 微小型毛细泵环热控制系统 及其制造技术 $[J]$. 机械工程学报, 2007, 43(12): 166-171.

CHI Yong, TANG Yong, CHEN Jinchang, et al. Miniaturized capillary pumped loop heat controlling system and it's manufacturing technigue[J]. Journal of Mechanical Engineering, 2007, 43(12): 166-171.

[5] HINZE B, ROSLER J. Measuring and simulating acoustic absorption of open-celled metals[J]. Advanced Engineering Materials, 2014, 16(3): 284-288.

[6] GAO Jun, DONG Xingfa, LIN Weiming. Selective catalytic methanation of $\mathrm{CO}$ in hydrogen-rich gas with a metal foam microreactor [J]. Journal of Fuel Chemistry and Technology, 2010, 38(3): 337-342.

[7] ZHOU Wei, KE Yuzhi, WANG Qinghui, et al. Development of cylindrical laminated methanol steam reforming microreactor with cascading metal foams as catalyst support[J]. Fuel, 2017, 191: 46-53.

[8] AARTUN I, SILBEROVA B, VENVIK H, et al. Hydrogen production from propane in Rh-impregnated metallic microchannel reactors and alumina foams[J]. Catalysis Today, 2005, 105(3-4): 469-478

[9] MUTH J, POGGIE M, KULESHA G, et al. Novel highly porous metal technology in artificial hip and knee replacement : Processing methodologies and clinical applications[J]. Journal of Metals, 2013， 65: 318-325.

[10] SCOTT M F, ALBERT S J, JUN Q. Investigation of the spark cycle on material removal rate in wire electrical discharge machining of advanced materials[J]. International Journal of Machine Tool \& Manufacture, 2004, 44: 391-400.

[11] ALEXANDER M M, DENNIS K, NORBERT J, et al. Machining of Metal Foams with varying mesostructure using Wire EDM[C]. Procedia CIRP, 2016, 42: 263-267.

[12] MISHRA D K, SARAVANAN T T, KHANRA G P, et al. Studies on the processing of nickel base porous wicks for capillary pumped loop for thermal management of spacecrafts[J]. Advanced Powder Technology, 2010, 21(6): 658-662.

[13] FUJIMURA T, NORIMATSU T, NAKAI M, et al. Laser machining of RF foam by second harmonics of Nd: YAG laser[J]. Fusion Science \& Technology, 2007, 51(4): 677-681.

[14] BAUMANN R, HERWIG P, WETZIG A, et al. Investigations of corrosion resistance of laser separated open cell metal[J]. Advanced Engineering Materials, 2017, 19(10): 1700107.

[15] LIU Yangxu, ZHOU Wei, Chu Xuyang, et al. Feasibility investigation of direct laser cutting process of metal foam with high pore density[J]. International Journal of Advanced Manufacturing Technology, 2018, 96(5-8): 2803-2814.

[16] SCHOOP J, AMBROSY F, ZANGER F, et al. Cryogenic machining of porous tungsten for enhanced surface integrity[J]. Journal of Materials Processing Technology, 2016, 229: 614-621.

[17] LIU Zhiqiang, HAN Jingjing, SU Yu, et al. Experimental investigation of porous metal materials in high-speed micro-milling process[J]. Proceedings of the Institution of Mechanical Engineers, Part B: Journal of Engineering Manufacture, 2018, 232(14): 2488-2498.

[18] BANHART J. Manufacture, characterization and application 
of cellular metals and metal foams[J]. Progress in Materials Science, 2001, 46(6): 559-632.

[19] SCHWINGELAD, SEELIGERAH W, VECCHIONACCIB C, et al. Aluminium foam sandwich structures for space application [J]. Acta Astronautica, 2007, 61: 1-6.

[20] ZHOU Wei, YU Wei, KE Yuzhi, et al. Size Effect and Series-parallel integration design of laminated methanol steam reforming microreactor for hydrogen production[J]. International Journal of Hydrogen Energy, 2018, 43(42): 19396-19404.

[21] TARTER J O, EFFGEN M, PUSAVEC F, et al. Cryogenic machining of porous tungsten for dispenser cathode applications[C]. Vacuum Electronics Conference, 2008. IVEC 2008. IEEE International. IEEE， 2008: 293-294.

[22] YILBAS B S, AKHTAR S, KELES O. Laser cutting of triangular blanks from thick aluminum foam plate: Thermal stress analysis and morphology[J]. Applied Thermal Engineering, 2014, 62(1): 28-36.

[23] ZHOW Wei, KE Yuzhi, WANG Qinghui, et al. Development of cylindrical laminated methanol steam reforming microreactor with cascading metal foams as catalyst support[J]. Fuel, 2017, 191: 46-53.

[24] BROMLEY B, HESSEL V, RENKEN A, et al. "Sandwich
Reactor" for heterogeneous catalytic processes: $\mathrm{N}_{2} \mathrm{O}$ decomposition as a case study[J]. Chemical Engineering \& Technology, 2008, 31(8): 1162-1169.

[25] RAHLI O, TADRIST L, MISCEVIC M, et al. Fluid flow through randomly packed monodisperse fibers: The kozeny-carman parameter analysis[J]. Journal of Fluids Engineering, 1997, 119(1): 188-192.

[26] CHEN S, HEAD D, EFFGEN M, et al. An investigation of sustained machining performance for controlled surface quality requirements in porous tungsten[J]. IEEE Transactions on Electron Devices, 2005, 52(5) : 903-908.

[27] BRAM M, KEMPMANN C, LAPTEV A, et al Investigations on the machining of sintered titanium foams utilizing face milling and peripheral grinding[J]. Advanced Engineering Materials, 2010， 5(6): 441-447.

[28] SCHOOP J, AMBROSY F, ZANGER F, et al. Cryogenic machining of porous tungsten for enhanced surface integrity[J]. Journal of Materials Processing Technology, 2016, 229: 614-621.

作者简介: 周伟(通信作者), 男, 1982 年出生, 教授, 博士研究生导师。 主要研究方向为精密制造技术、新能源与节能技术、微纳传感器。

E-mail: weizhou@xmu.edu.cn 